\title{
The importance of mitochondrial folate enzymes in human colorectal cancer
}

\author{
MASAAKI MIYO $^{1}$, MASAMITSU KONNO $^{2}$, HUGH COLVIN $^{1,3}$, NAOHIRO NISHIDA $^{2}$, JUN KOSEKI $^{1}$, \\ KOICHI KAWAMOTO ${ }^{1,2}$, KENTA TSUNEKUNI ${ }^{1,3,4}$, JUNICHI NISHIMURA ${ }^{1}$, TAISHI HATA ${ }^{1}$, \\ ICHIRO TAKEMASA $^{1}$, TSUNEKAZU MIZUSHIMA ${ }^{1}$, YUICHIRO DOKI ${ }^{1}$, MASAKI MORI ${ }^{1}$ and HIDESHI ISHII ${ }^{2,3}$ \\ Departments of ${ }^{1}$ Gastroenterological Surgery, ${ }^{2}$ Frontier Science for Cancer and Chemotherapy, \\ ${ }^{3}$ Cancer Profiling Discovery, Osaka University Graduate School of Medicine, Suita, Osaka 565-0871; \\ ${ }^{4}$ Taiho Pharmaceutical Co., Ltd., Chiyoda-ku, Tokyo 101-0054, Japan
}

Received June 3, 2016; Accepted October 17, 2016

DOI: $10.3892 /$ or.2016.5264

\begin{abstract}
Folate plays a pivotal role in the one-carbon metabolism needed for methylation reactions, nucleotide synthesis, and DNA repair. Although folate metabolism was recently shown to be associated with carcinogenesis in some solid tumors, the importance of folate metabolism in colorectal cancer remains unclear. In the present investigation we found that expression of three mitochondrial folate metabolic enzymes, serine hydroxymethyl transferase (SHMT2), methylenetetrahydrofolate dehydrogenase (MTHFD2) and aldehyde dehydrogenase 1 family member L2 (ALDH1L2), were upregulated in human colorectal tumor tissues compared to normal tissues. Colorectal cancer tissue samples were obtained from 117 consecutive patients. We evaluated the expression of the enzymes with immunohistochemical analysis and determined their relevance to clinicopathological characteristics and prognosis. Rates of recurrence-free survival (RFS) and overall survival (OS) in patients with high expression of SHMT2, MTHFD2 and ALDH1L2 tended to be lower than in patients with low expression of SHMT2, MTHFD2 and ALDH1L2 ( $\mathrm{P}=0.446$ and $\mathrm{P}=0.337, \mathrm{P}=0.099$ and $\mathrm{P}=0.064$, $\mathrm{P}=0.178$ and $\mathrm{P}=0.257$, respectively). Notably, the combined high expression of SHMT2, MTHFD2 and ALDH1L2 (triple high) was more highly associated with poor prognosis than the individual expression levels (RFS; $\mathrm{P}=0.004$ and OS; $\mathrm{P}=0.037)$. A multivariate analysis showed that triple high expression was independently associated with RFS ( $\mathrm{P}=0.017)$. These findings suggested that mitochondrial folate metabolic
\end{abstract}

Correspondence to: Professor Hideshi Ishii, Department of Cancer Profiling Discovery, Osaka University Graduate School of Medicine, Yamadaoka 2-2, Suita, Osaka 565-0871, Japan

E-mail: hishii@gesurg.med.osaka-u.ac.jp

Key words: serine hydroxymethyl transferase, methylenetetrahydrofolate dehydrogenase, aldehyde dehydrogenase 1 family member L2, colorectal cancer, folate enzymes could provide a potential therapeutic strategy for treating colorectal cancer.

\section{Introduction}

Tumor tissues are different from normal tissues in many aspects, mainly due to differences in vasculature (1). In tumor tissues, immature vasculature forms a microenvironment of hypoxia and malnutrition (2). Tumor cells change their metabolism to be able to survive and proliferate in these harsh conditions. High rates of glucose consumption and lactate production, even in the presence of oxygen, have been observed in various tumor cells; this phenomenon is known as the Warburg effect (3). Since Warburg's study, the metabolism of normal and tumor tissues has been studied, and differences are expected to serve as a basis for new therapeutic targets for refractory cancers.

Folate plays a pivotal role in the one-carbon metabolism needed for methylation reactions, nucleotide synthesis, and DNA repair $(4,5)$. Methylation of DNA and histones is a well known epigenetic mechanism, and it is the most common molecular alteration in cancer cells (6). Furthermore, cells with high rates of proliferation, like tumor cells and embryogenic cells, require the synthesis of abundant proteins, lipids and nucleotides (7). Due to these features, it was proposed that folate may be associated with carcinogenesis (8). Jain et al reported that expression of the mitochondrial glycine biosynthetic pathway was significantly correlated with cancer cell proliferation, based on the consumption and release of 219 metabolites from NCI-60 cancer cell lines. Moreover, high expression levels of three mitochondrial glycine (also folate) metabolism enzymes, serine hydroxymethyl transferase (SHMT2), methylenetetrahydrofolate dehydrogenase (MTHFD2) and tetrahydrofolate synthase (MTHFD1L), were associated with greater mortality in patients with breast cancer (9). The expression of mitochondrial folate metabolic enzymes was reported to be a determinant of the response to methotrexate, and the dihydrofolate reductase inhibitor is used most often as an anticancer drug in clinical settings (10). Although previous studies have shown that mitochondrial folate metabolic enzymes correlated with some types of solid tumors, their importance in colorectal cancer remains unclear (11). 
The aim of the present study was to assess the importance of mitochondrial folate metabolic enzymes in human colorectal cancer. Our results indicated that expression levels of mitochondrial folate metabolic enzymes, including SHMT2, MTHFD2 and aldehyde dehydrogenase 1 family member L2 (ALDH1L2) were upregulated in human colorectal tumor tissues compared to normal tissues. Moreover, immunohistochemical analysis showed that the combined high expression of SHMT2, MTHFD2 and ALDH1L2 (triple high expression) was more highly associated with a poor prognosis than the individual expression levels. Moreover, a multivariate analysis indicated that triple high expression was independently associated with recurrence-free survival. We concluded that mitochondrial folate metabolic enzymes could provide a potential therapeutic strategy against colorectal cancer.

\section{Materials and methods}

The Cancer Genome Atlas tissue samples. The colorectal adenocarcinoma data were generated by the Cancer Genome Atlas (TCGA) Research Network: http://cancergenome.nih.gov/. Messenger RNA expression and DNA methylation were compared between normal colorectal tissue and cancer with Wanderer software (12). We analyzed exon transcript values ( $\log _{2}$ RPKM) of chr12:57624586-57624783 for SHMT2, chr2:74425690-74425869 for MTHFD2 and chr12:105413564-105418257 for ALDH1L2. The mean methylation $\beta$ values of all the HumanMethylation 450 probes inside the genes were evaluated. The mutation frequency was assessed in 220 colorectal tumor samples with sequencing data through the cBioPortal for Cancer Genomics (http://cbioportal.org) $(13,14)$.

Prognosis of patients with colorectal cancer. The published GSE17536 database (http://www.ncbi.nlm.nih.gov/geo/query/ acc.cgi?acc=GSE17536), which included microarray data for 177 patients, was used to screen for genes related to the prognosis of patients with colorectal cancer, as described previously (15). The patient data were divided into two groups (low- and high-expression groups) based on the mean levels of SHMT2, MTHFD2 and ALDH1L2 expression. Then, we evaluated the relevance of these gene expression levels to overall survival.

Tissue samples. Colorectal cancer tissue samples were obtained from 117 consecutive patients that underwent surgery at the Osaka University Hospital, between 2006 and 2009. Written informed consent for the use of resected specimens was provided by all patients. The present study was approved by the Institutional Review Board (the approved protocol numbers were \#08226 and \#213). None of the patients had received chemotherapy or radiotherapy before surgery. A total of 108 patients underwent curative surgery and 9 patients underwent palliative surgery. Samples were fixed in buffered formalin at $4^{\circ} \mathrm{C}$ overnight, processed through graded ethanol solutions, and embedded in paraffin. Medical and pathology studies were reviewed to collect information regarding various clinicopathological parameters, including age, gender, carcinoembryonic antigen (CEA), carbohydrate antigen 19-9 (CA-19-9), histological type, depth of tumor invasion, lymph node metastasis, distant metastasis, pTNM stage (according to the TNM Classification System of Malignant Tumors, Seventh Edition) (16), venous invasion and lymphatic invasion.

Immunohistochemical analysis. Immunohistochemical analysis was performed with the Vectastain Elite ABC kit (Vector Laboratories, Burlingame, CA, USA) as previously described (17). Briefly, tissue sections (3.5 $\mu \mathrm{m}$ thick) were sliced from paraffin-embedded tissue blocks. After deparaffinization in xylene and dehydration in graded ethanol solutions, tissue sections were heated at $110^{\circ} \mathrm{C}$ for $15 \mathrm{~min}$ in $10 \mathrm{mM}$ citrate buffer ( $\mathrm{pH}$ 6.0), followed by incubation with $0.3 \%$ hydrogen peroxide for $20 \mathrm{~min}$ to block endogenous peroxidase activity. Subsequently, the sections were incubated with SHMT2 antibody (ab64417; 1:300 dilution; rabbit polyclonal), MTHFD2 antibody (ab176016; 1:100 dilution; rabbit polyclonal) and ALDH1L2 antibody (ab170176; 1:200 dilution; rabbit polyclonal) (all from Abcam, Cambridge, MA, USA) at $4^{\circ} \mathrm{C}$ overnight. Then, sections were incubated with a biotinylated secondary antibody solution for $30 \mathrm{~min}$, followed by incubation with $\mathrm{ABC}$ reagent for $30 \mathrm{~min}$. After development with diaminobenzidine, sections were counterstained with hematoxylin. As a negative control, phosphate-buffered saline was used instead of the antibodies. Representative positive colorectal cancer tissues were used as a positive control. The analyses were performed independently, in a blinded manner, by two pathologists.

Evaluation of immunostaining. The staining score was defined as the intensity of staining that covered the largest region. We first performed immunostaining for 20 colorectal cancer samples randomly chosen to decide the concentration of antibodies and samples that could be applied for a positive control. The samples showing an average staining were used as a positive control. Unstained tissues were assigned to 'negative staining (-)'. The tissues stained weaker than the positive control but stronger than the unstained tissue were categorized into 'weak staining (+)' and the tissues stained stronger than the positive control were categorized into 'strong staining (++)'. To evaluate associations between expression levels and clinicopathological features or survival, the samples were divided into two groups, as follows: low expression (-) and high expression $(+$ and ++ ) for SHMT2 and ALDH1L2, or low expression (- and + ) and high expression (++) for MTHFD2.

Statistical analysis. Data were expressed as the mean \pm standard deviation (SD). We performed statistical analyses with JMP Pro 10 software (SAS Institute, Cary, NC, USA). Statistically significant differences were determined with Fisher's exact test. Recurrence-free survival and overall survival were estimated with the Kaplan-Meier method, and the statistical significance was evaluated with the log-rank test. Variables identified as significant in univariate analyses were entered into the Cox proportional hazards regression models. A P-value $<0.05$ was taken to indicate a significant difference.

\section{Results}

Upregulation of enzymes associated with mitochondrial folate metabolic pathway in colorectal cancer. Reportedly, enzymes 

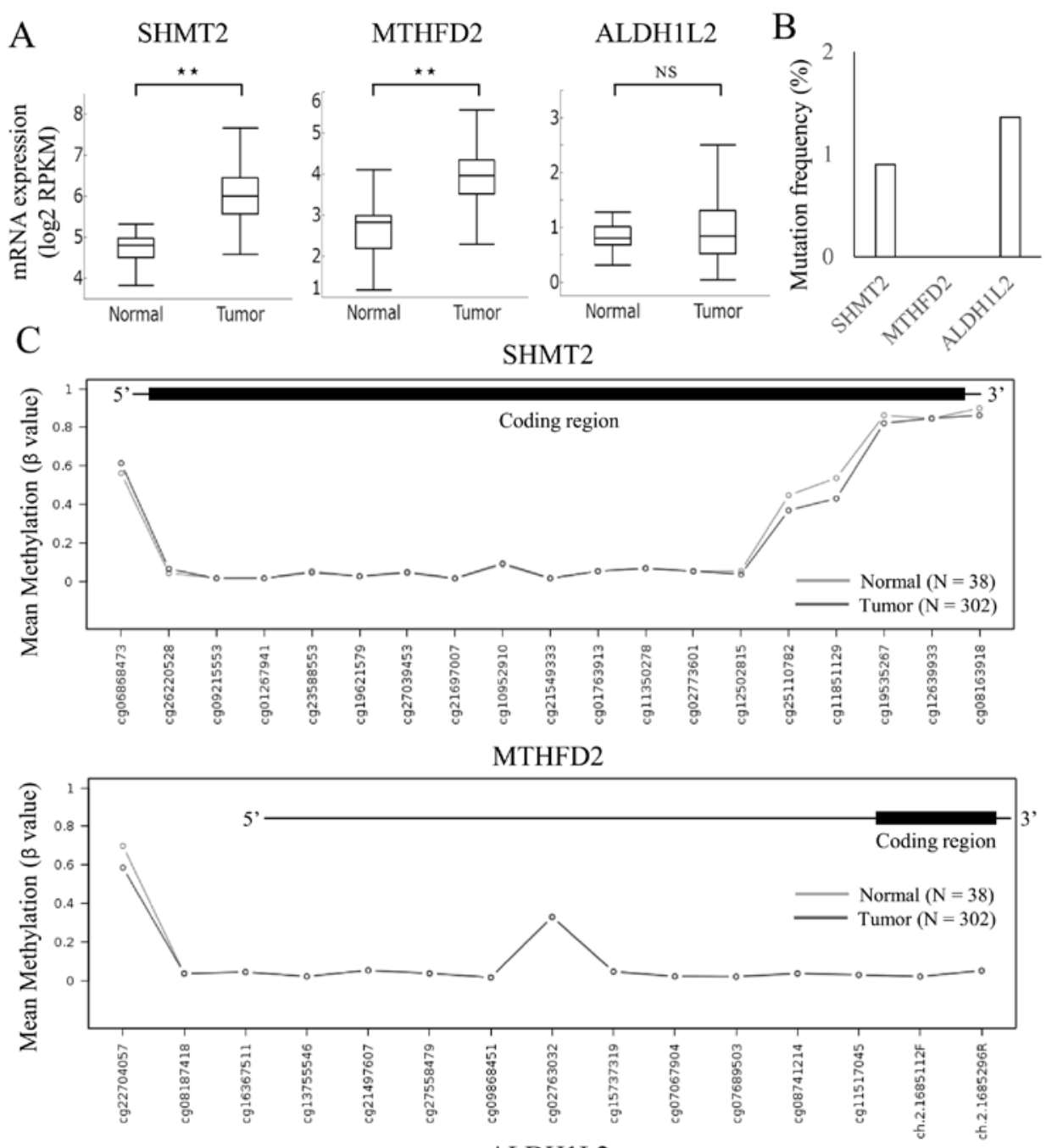

ALDH1L2

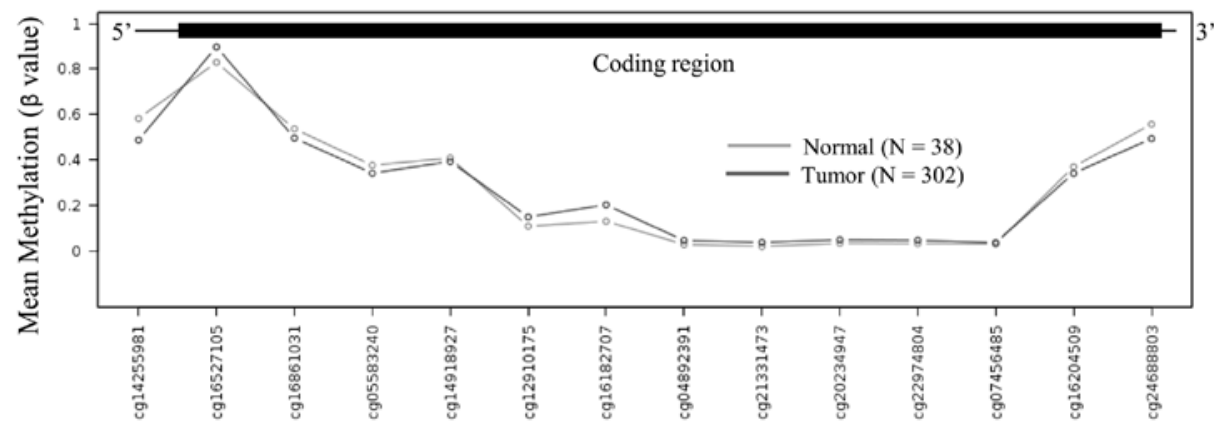

Figure 1. Upregulation of enzymes associated with the mitochondrial folate metabolic pathway in colorectal tumor tissue. (A) Exon transcript values $\left(\log _{2}\right.$ RPKM) of SHMT2, MTHFD2 and ALDH1L2 in normal $(n=41)$ and tumor colorectal tissues $(n=262)$. (B) Mean mutation frequency in 220 colorectal tumor samples. (C) The mean $\beta$ values for SHMT2, MTHFD2 and ALDH1L2 DNA methylation in normal ( $\mathrm{n}=38$ ) and tumor colorectal tissues ( $\mathrm{n}=302$ ). The results shown here are wholly based on data generated by the TCGA Research Network. ( ${ }^{* *}$ P $<0.01$; NS, not significant). SHMT2, serine hydroxymethyl transferase; MTHFD2, methylenetetrahydrofolate dehydrogenase; ALDH1L2, aldehyde dehydrogenase 1 family member L2.

associated with the mitochondrial folate metabolic pathway show upregulated expression in cancer (18). To confirm expression in normal colorectal tissue and colorectal cancer, we analyzed the data from the TCGA Research Network. We found that the mRNA expression levels of SHMT2, MTHFD2 and ALDH1L2 associated with mitochondrial folate metabolism were higher in colorectal cancer than in normal colorectal tissue $(\mathrm{P}<0.001, \mathrm{P}<0.001$ and $\mathrm{P}=0.681$, respectively) (Fig. $1 \mathrm{~A})$. Several studies have indicated that gene mutations change the activity and function of metabolic enzymes. For example, mutations in isocitrate dehydrogenase 1 resulted in a new enzymatic function: catalysis of the NADPH-dependent reduction of $\alpha$-ketoglutarate to R(-)-2-hydroxyglutarate (19). A mutation in mitochondrial aldehyde dehydrogenase 2 , which is essential for alcohol detoxification, induced structural alterations that changed its enzymatic activity (20). Therefore, we assessed the importance of mutations in SHMT2, MTHFD2 and ALDH1L2 in colorectal tumor samples. The mutation frequency was $<2 \%$ (Fig. 1B). Methylation of these genes was also evaluated, based on the data from the TCGA Research 

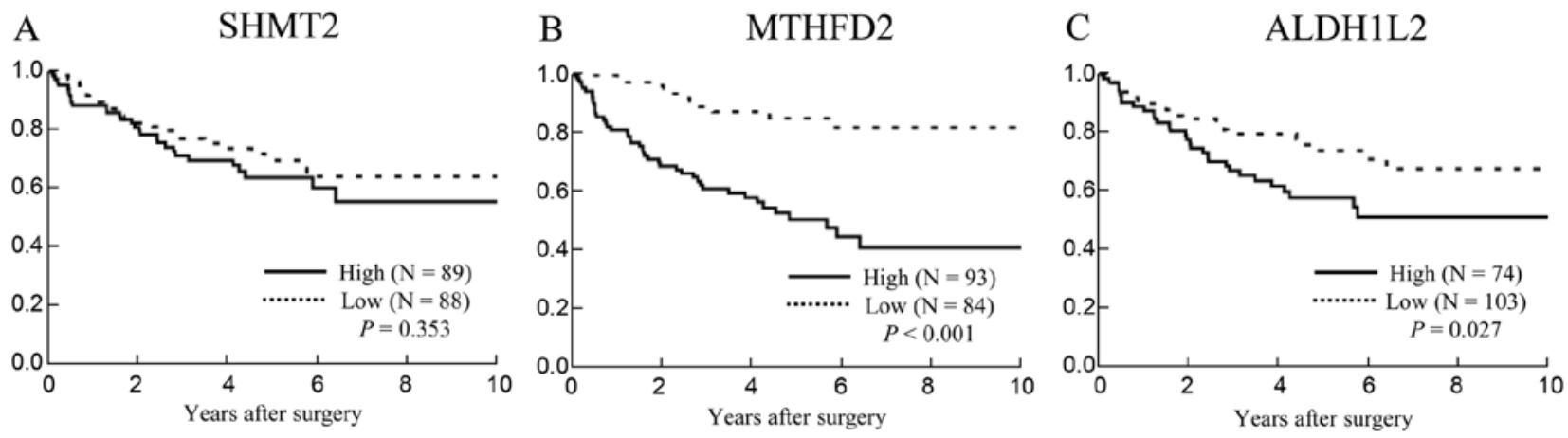

Figure 2. Kaplan-Meier curves show overall survival. Survival is shown according to (A) SHMT2, (B) MTHFD2 and (C) ALDH1L2 mRNA expression levels. Differences between high and low expression groups were evaluated with the log-rank test. The results are based on data from the GSE17536 microarray database. Ordinate, survival rate; abscissa, years after surgery. SHMT2, serine hydroxymethyl transferase; MTHFD2, methylenetetrahydrofolate dehydrogenase; ALDH1L2, aldehyde dehydrogenase 1 family member L2.

A

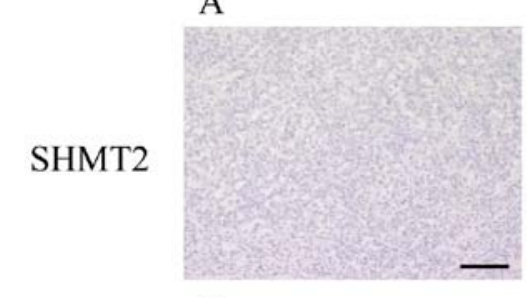

D

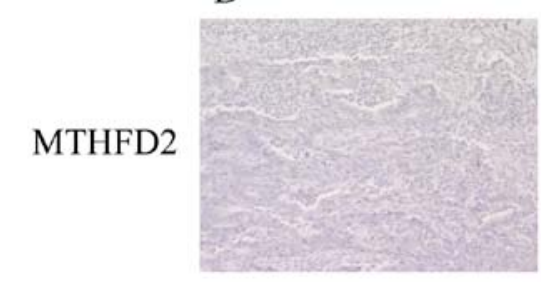

G

ALDH1L2
B

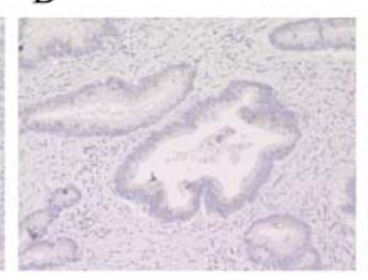

E

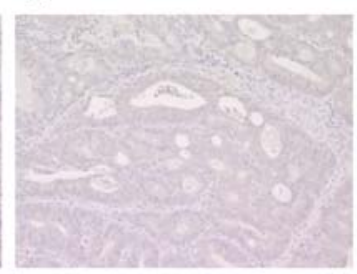

$\mathrm{H}$

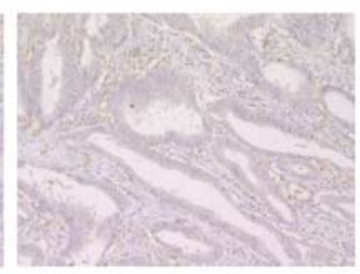

$\mathrm{C}$

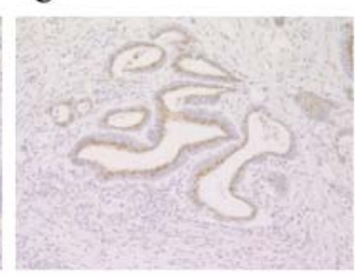

F

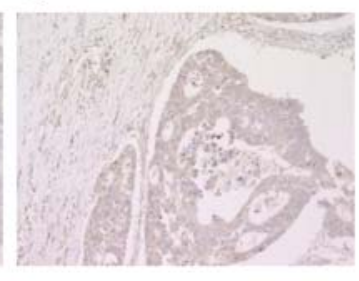

I

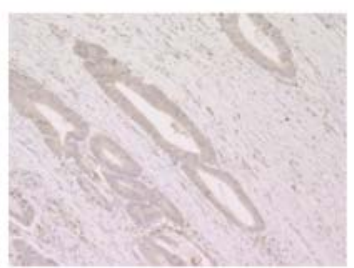

Figure 3. Immunohistochemical detection of protein expression. (A-C) SHMT2, (D-F) MTHFD2 and (G-I) ALDH1L2 expression levels varied in colorectal cancer tissues. Examples are shown for (A, D and G) negative staining (-), (B, E and H) weak staining (+), and (C, F and I) strong staining (++). Scale bar indicates, $100 \mu \mathrm{m}$. SHMT2, serine hydroxymethyl transferase; MTHFD2, methylenetetrahydrofolate dehydrogenase; ALDH1L2, aldehyde dehydrogenase 1 family member L2.

Network. The methylation frequency was similar in normal and tumor colorectal tissues (Fig. 1C). These findings showed that expression of SHMT2, MTHFD2 and ALDH1L2 was associated with the physiological characteristics of colorectal cancer.

Relevance of SHMT2, MTHFD2 and ALDH1L2 mRNA expression in colorectal cancer with respect to prognosis. Previous studies showed a correlation between the expression levels of mitochondrial folate metabolism enzymes and the prognosis of some solid tumors. Therefore, we analyzed microarray data from patients with colorectal cancer in the GSE17536 database $(11,21)$. The overall survival rate in patients with high expression levels of MTHFD2 and ALDH1L2 was significantly lower than in patients with low expression levels of MTHFD2 and ALDH1L2 ( $\mathrm{P}<0.001$ and $\mathrm{P}=0.027$, respectively) (Fig. 2). We also found a trend for lower overall survival rate in patients with high expression of SHMT2 compared to patients with low expression of SHMT2 ( $\mathrm{P}=0.353)$.

Immunohistochemistry for SHMT2, MTHFD2 and ALDH1L2 in colorectal cancer. To study the importance of SHMT2, MTHFD2 and ALDH1L2 further, we collected colorectal cancer samples and analyzed the expression of SHMT2, MTHFD2 and ALDH1L2 at protein level with immunohistochemistry. The median follow-up duration of patients with colorectal cancer was 4.9 years (range; $0.2-6.6$ years). SHMT2, MTHFD2 and ALDH1L2 expression was observed in the 
Table I. Immunohistochemical detection results for SHMT2, MTHFD2 and ALDH1L2 in colorectal cancer.

\begin{tabular}{|c|c|c|c|c|c|}
\hline Clinicopathological factors & Classification & $\mathrm{N}$ & $\begin{array}{l}\text { Triple high } \\
\text { (N) }\end{array}$ & $\begin{array}{l}\text { Others } \\
(\mathrm{N})\end{array}$ & P-value \\
\hline \multicolumn{6}{|l|}{ Patient background } \\
\hline \multirow[t]{2}{*}{ Gender } & Male & 71 & 17 & 54 & 0.829 \\
\hline & Female & 46 & 12 & 34 & \\
\hline \multirow[t]{2}{*}{ Age } & $<65$ & 57 & 16 & 41 & 0.522 \\
\hline & $\geq 65$ & 60 & 13 & 47 & \\
\hline \multirow[t]{2}{*}{ CEA } & $<3.1$ & 66 & 16 & 50 & 1.000 \\
\hline & $\geq 3.1$ & 51 & 13 & 50 & \\
\hline \multirow{2}{*}{ CA19-9 } & $<40$ & 101 & 23 & 78 & 0.221 \\
\hline & $\geq 40$ & 16 & 6 & 10 & \\
\hline \multicolumn{6}{|l|}{ Tumor characteristics } \\
\hline \multirow{2}{*}{ Histological type $\mathrm{a}^{\mathrm{a}}$} & tub1, tub2, pap & 109 & 26 & 83 & 0.407 \\
\hline & por, muc & 8 & 3 & 5 & \\
\hline \multirow{2}{*}{ Depth of tumor invasion } & Tis, T1, T2 & 49 & 11 & 38 & 0.669 \\
\hline & $\mathrm{T} 3, \mathrm{~T} 4$ & 68 & 18 & 50 & \\
\hline \multirow[t]{2}{*}{ Lymph node metastasis } & Positive & 49 & 13 & 36 & 0.829 \\
\hline & Negative & 68 & 16 & 52 & \\
\hline \multirow[t]{2}{*}{ Distant metastasis } & Positive & 13 & 4 & 9 & 0.734 \\
\hline & Negative & 104 & 25 & 79 & \\
\hline \multirow[t]{2}{*}{ Lymphatic invasion } & Positive & 85 & 22 & 63 & 0.811 \\
\hline & Negative & 32 & 7 & 25 & \\
\hline \multirow[t]{2}{*}{ Venous invasion } & Positive & 29 & 8 & 21 & 0.805 \\
\hline & Negative & 88 & 21 & 67 & \\
\hline \multirow[t]{2}{*}{ Stage } & 0, I, II & 62 & 14 & 48 & 0.669 \\
\hline & III, IV & 55 & 15 & 40 & \\
\hline
\end{tabular}

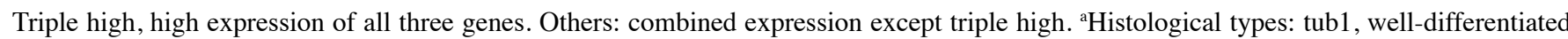
adenocarcinoma; tub2, moderately differentiated adenocarcinoma; pap, papillary adenocarcinoma; por, poorly differentiated adenocarcinoma; muc, mucinous carcinoma.

glandular cells of colorectal cancer (Fig. 3). Fig. 3B, E and H shows the samples that were used as a positive control. Subsequently, associations between the expression levels of these genes and prognosis were evaluated. Rates of recurrencefree and overall survival in patients with high expression of SHMT2, MTHFD2 and ALDH1L2 tended to be lower than in patients with low expression of SHMT2, MTHFD2 and ALDH1L2, but these differences were not significant $(\mathrm{P}=0.446$ and $\mathrm{P}=0.337, \mathrm{P}=0.099$ and $\mathrm{P}=0.064, \mathrm{P}=0.178$ and $\mathrm{P}=0.257$, respectively) (Fig. 4A-C). Notably, high expression of SHMT2, MTHFD2 and ALDH1L2 (triple high) was more highly associated with poor prognosis than increased expression levels of each individual gene (Fig. 4D). These results indicated that malignancy in colorectal cancer was influenced by activation of the mitochondrial folate metabolic pathway.

Impact of triple high expression on recurrence-free survival. Based on our findings that the expression levels of enzymes associated with the mitochondrial folate metabolic pathway could influence colorectal cancer malignancy, we assessed the relevance of the expression of three mitochondrial folate enzymes to clinicopathological features. There were no significant association between the individual expression of MTHFD2 and ALDH1L2 and patient and tumor characteristics (data not shown). In regards to SHMT2, its expression was negatively associated with lymph node metastasis, lymphatic invasion and stage $(\mathrm{P}=0.015, \mathrm{P}=0.021$ and $\mathrm{P}=0.010$, respectively, data not shown). Interestingly, triple high expression did not significantly correlate with patient background characteristics, including gender, age, CEA and CA19-9; or with tumor characteristics, including histological type, depth of tumor invasion, lymph node metastasis, distant metastasis, lymphatic invasion, venous invasion and stage (Table I). To determine associations between triple high expression and the most important factors $(\mathrm{P}<0.100)$, we included depth of tumor invasion, lymph node metastasis, lymphatic invasion, venous invasion, stage, and SHMT2, MTHFD2 and ALDH1L2 expression in a multivariate analysis for recurrence-free survival (Table II). This multivariate analysis showed that triple high expression was only independently associated with recurrence-free survival $(\mathrm{P}=0.017)$

\section{Discussion}

We found that expression levels of the mitochondrial folate metabolic enzymes, SHMT2, MTHFD2 and ALDH1L2, were upregulated in colorectal tumor tissues and that triple high expression was associated with a poor prognosis. The folate 
A

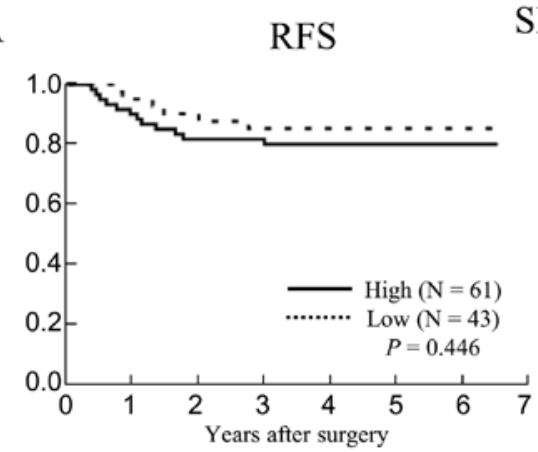

B

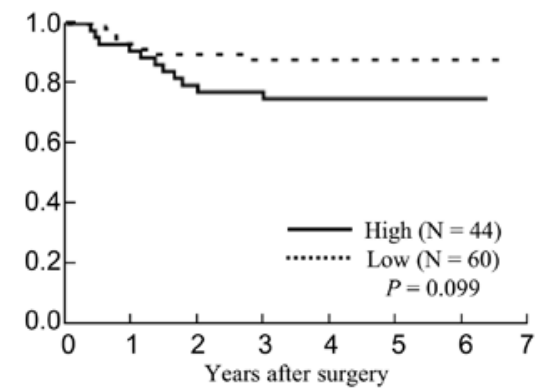

SHMT2

OS

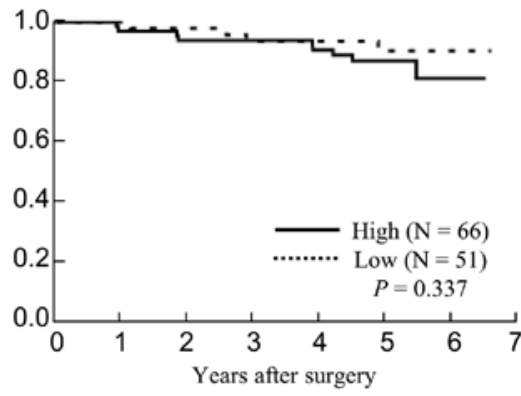

MTHFD2

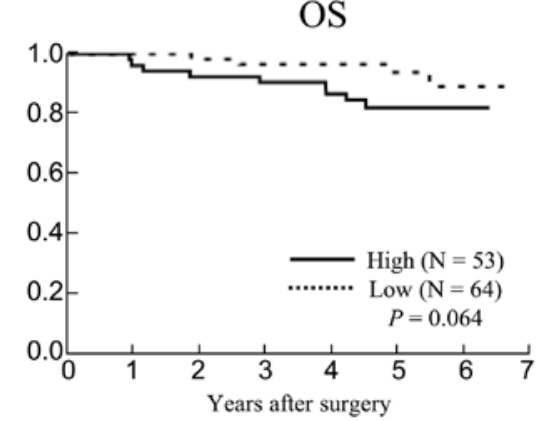

ALDH1L2 OS
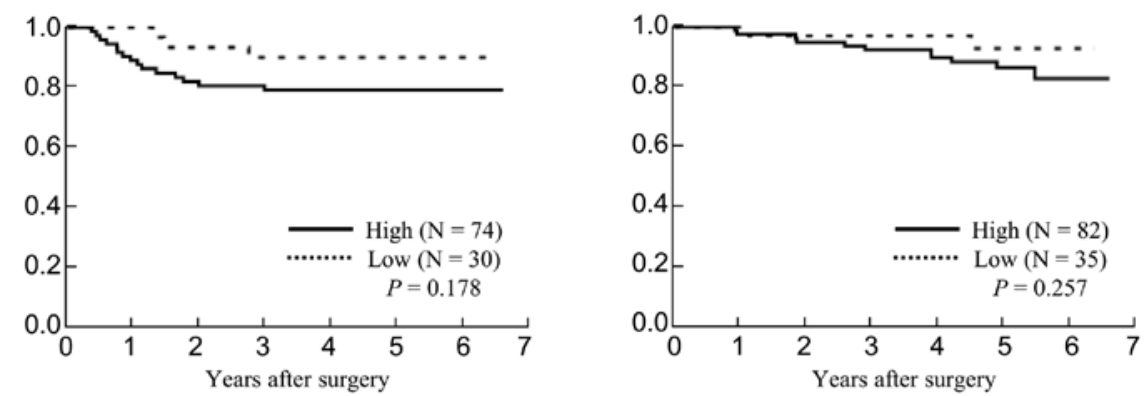

RFS Triple high vs others

OS

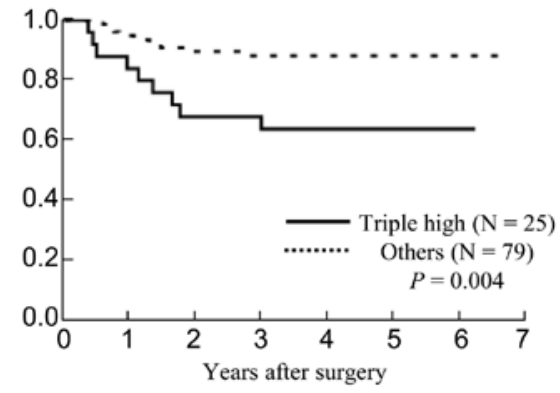

Figure 4. Kaplan-Meier curves show RFS and OS. Survival is shown according to high and low expression of (A) SHMT2, (B) MTHFD2 and (C) ALDH1L2. (D) The survival of patients with combined high expression of all three genes (triple high expression) compared to other combined expression except triple high. Differences between groups were evaluated with the log-rank test. Ordinate, survival rate; abscissa, years after surgery. SHMT2, serine hydroxymethyl transferase; MTHFD2, methylenetetrahydrofolate dehydrogenase; ALDH1L2, aldehyde dehydrogenase 1 family member L2; RFS, recurrence-free survival; OS, overall survival.

metabolic pathway comprises a cycle mediated by SHMT2, MTHFD2 and ALDH1L2 (Fig. 5). Our results indicated that activation of the mitochondrial folate metabolic pathway without stagnation could offer advantages for survival and proliferation in colorectal cancer. SHMT2 negatively regulates the activity of pyruvate kinase, and subsequently, it decreases carbon flux into the TCA cycle; this activity provides a survival benefit for glioma cells under hypoxic conditions (22). An analysis of 515 tumor-derived cell lines showed that patients with significant upregulation of folate metabolism genes tended to benefit from treatment with methotrexate (10). In addition to its importance in methylation reactions, nucleotide synthesis, and DNA repair, the mitochondrial folate metabolic pathway also influences the biological characteristics of tumors, which contribute to tumor malignancy. Interfering with activation of the mitochondrial folate metabolic pathway could be a potential therapeutic strategy for treating refractory colorectal cancer. 
Table II. Univariate and multivariate analyses of recurrence-free survival.

\begin{tabular}{|c|c|c|c|c|c|c|}
\hline \multirow[b]{2}{*}{ Clinicopathological factors } & \multirow[b]{2}{*}{ Classification } & \multirow[b]{2}{*}{$\mathrm{N}$} & \multicolumn{2}{|c|}{ Univariate analysis } & \multicolumn{2}{|c|}{ Multivariate analysis } \\
\hline & & & $\mathrm{HR}^{\mathrm{a}}$ & P-value & $\mathrm{HR}^{\mathrm{a}}$ & P-value \\
\hline \multicolumn{7}{|l|}{ Patient background } \\
\hline \multirow[t]{2}{*}{ Gender } & Male & 65 & 1.69 & 0.305 & & \\
\hline & Female & 39 & $(0.64-5.25)$ & & & \\
\hline \multirow[t]{2}{*}{ Age } & $<65$ & 47 & 1.51 & 0.384 & & \\
\hline & $\geq 65$ & 57 & $(0.59-3.95)$ & & & \\
\hline \multirow[t]{2}{*}{ CEA } & $<3.1$ & 62 & 2.07 & 0.123 & & \\
\hline & $\geq 3.1$ & 42 & $(0.82-5.43)$ & & & \\
\hline \multirow[t]{2}{*}{ CA19-9 } & $<40$ & 93 & 1.26 & 0.766 & & \\
\hline & $\geq 40$ & 11 & $(0.20-4.43)$ & & & \\
\hline \multicolumn{7}{|l|}{ Tumor characteristics } \\
\hline \multirow[t]{2}{*}{ Histological type ${ }^{\mathrm{b}}$} & tub1, tub2, pap & 97 & 1.26 & 0.817 & & \\
\hline & por, muc & 7 & $(0.26-22.66)$ & & & \\
\hline \multirow[t]{2}{*}{ Depth of tumor invasion } & Tis, $\mathrm{T} 1, \mathrm{~T} 2$ & 49 & 3.59 & 0.013 & 1.97 & 0.297 \\
\hline & $\mathrm{T} 3, \mathrm{~T} 4$ & 55 & $(1.29-12.68)$ & & $(0.57-8.25)$ & \\
\hline \multirow[t]{2}{*}{ Lymph node metastasis } & Positive & 38 & 3.17 & 0.015 & 1.03 & 0.977 \\
\hline & Negative & 66 & $(1.25-8.63)$ & & $(0.17-19.66)$ & \\
\hline \multirow[t]{2}{*}{ Lymphatic invasion } & Positive & 73 & 3.88 & 0.033 & 1.51 & 0.629 \\
\hline & Negative & 31 & $(1.10-24.55)$ & & $(0.30-11.17)$ & \\
\hline \multirow[t]{2}{*}{ Venous invasion } & Positive & 22 & 3.14 & 0.027 & 1.80 & 0.291 \\
\hline & Negative & 82 & $(1.15-7.99)$ & & $(0.60-5.32)$ & \\
\hline \multirow[t]{2}{*}{ Stage } & $0, \mathrm{I}, \mathrm{II}$ & 62 & 3.43 & 0.011 & 1.66 & 0.672 \\
\hline & III & 42 & $(1.33-9.86)$ & & $(0.08-11.92)$ & \\
\hline \multirow{2}{*}{$\begin{array}{l}\text { SHMT2, MTHFD2 } \\
\text { and ALDH1L2 }\end{array}$} & Triple high & 25 & 3.55 & 0.009 & 3.21 & 0.017 \\
\hline & Others & 79 & $(1.39-9.11)$ & & $(1.24-8.31)$ & \\
\hline
\end{tabular}

${ }^{a} \mathrm{HR}$, hazard ratio (95\% confidence interval); ${ }^{b}$ histological types: tub1, well-differentiated adenocarcinoma; tub2, moderately differentiated adenocarcinoma; pap, papillary adenocarcinoma; por, poorly differentiated adenocarcinoma; muc, mucinous carcinoma; ${ }^{c}$ expression levels: triple high, high expression of all three genes. Others: combined expression except triple high.

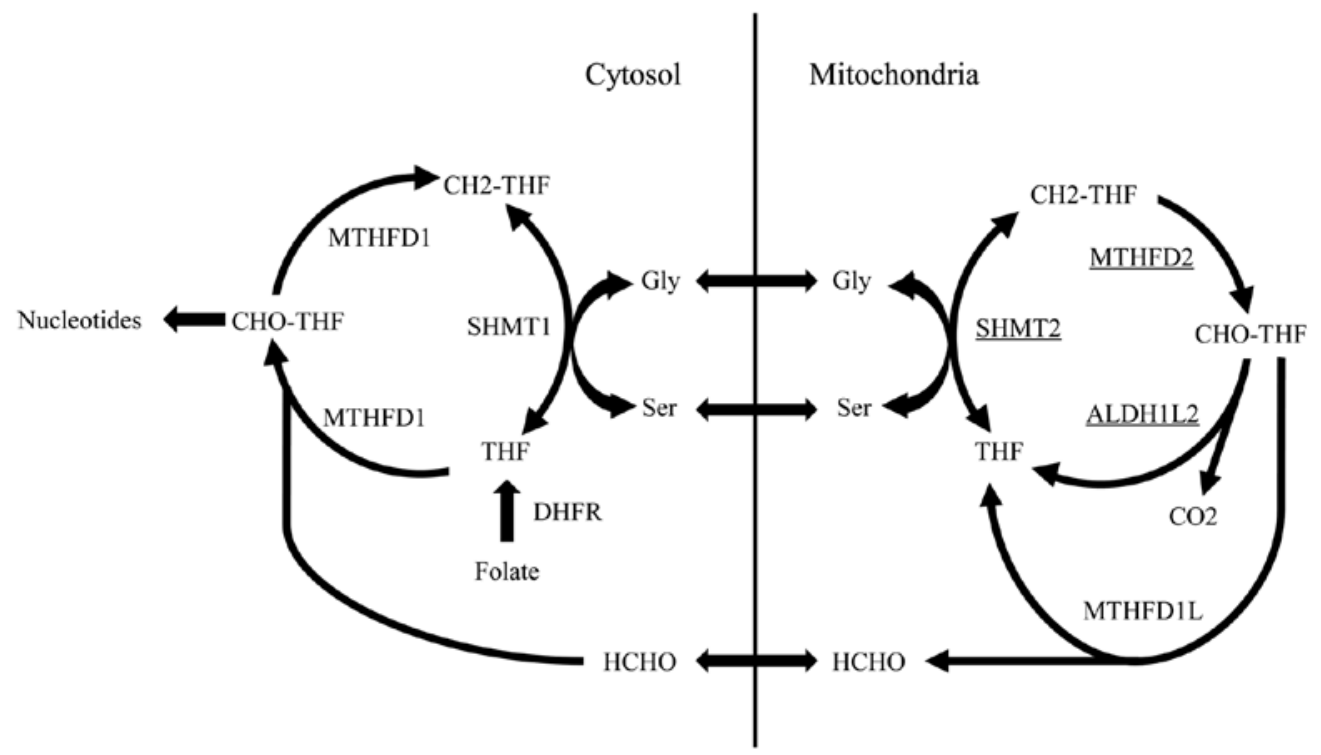

Figure 5. Schematic overview of the folate metabolic pathway mediated by SHMT2, MTHFD2 and ALDH1L2. (Left) Cytosolic folate metabolism; (right) mitochondrial folate metabolism. Arrows that cross the line indicate metabolites that can travel between compartments. ALDH1L2, aldehyde dehydrogenase 1 family member L2; CH2-THF, methylenetetrahydrofolate; CHO-THF, formyl-tetrahydrofolate; DHFR, dihydrofolate reductase; Gly, glycine; MTHFD1, methylenetetrahydrofolate dehydrogenase 1; MTHFD1L, methylenetetrahydrofolate dehydrogenase 1-like; MTHFD2, methylenetetrahydrofolate dehydrogenase 2; HCHO, formaldehyde; Ser, serine; SHMT1, serine hydroxymethyltransferase 1; SHMT2, serine hydroxymethyltransferase 2; THF, tetrahydrofolate. 
Reportedly, only mitochondrial, not cytosolic, folate enzymes were significantly associated with rapid cancer cell proliferation and prognosis in breast cancer (9). To elucidate the importance of folate enzymes, including dihydrofolate reductase, MTHFD1 and SHMT1, the prognosis of patients with colorectal cancer was assessed based on data from the published GSE17536 database. Rates of disease-free and overall survival were not significantly different between groups with high and low dihydrofolate reductase expression $(\mathrm{P}=0.290$ and $\mathrm{P}=0.906$, respectively) or between groups with high and low MTHFD1 expression $(\mathrm{P}=0.252$ and $\mathrm{P}=0.279$, respectively) (data not shown). Conversely, low SHMT1 expression was significantly associated with a short overall survival rate $(\mathrm{P}=0.033)$ (data not shown). These data supported the hypothesis that mitochondrial folate metabolism may be more important than cytosolic folate metabolism in human colorectal cancer. The detailed function and mechanism of cytosolic folate metabolism in tumor cells remain unclear. Additional studies are needed to address whether the function of cytosolic folate enzymes differs from that of mitochondrial folate enzymes.

Few studies have described the regulation of mitochondrial folate enzymes. Reportedly, mammalian target of rapamycin complex 1 (mTORC1) positively regulates the expression of MTHFD2 via activating transcription factor 4 (23). It is well known that activation of mTORC1 is correlated with nutrient, energy and redox sensing processes (24). Therefore, the expression of mitochondrial folate enzymes may also be regulated by environmental factors, including oxidative stress, growth factors and amino acids. mTORC1 controls several anabolic metabolism pathways required for cell proliferation and growth. This function is thought to depend partially on mitochondrial folate metabolism, which is associated with nucleotide synthesis, because nucleotides are necessary for cell proliferation and growth.

The formyl-tetrahydrofolate synthase activity of MTHFD1L was reported to play an important role in the proliferation of breast cancer cells (9). MTHFD1L also participates in the cycle of the mitochondrial folate pathway (Fig. 5). We assessed the relationship between MTHFD1L expression and prognosis of patients with colorectal cancer, based on data from the GSE17536 database. However, we found that MTHFD1L expression was not significantly related to the prognosis of patients (data not shown). Although ALDH1L2 and MTHFD1L catalyze the same reaction, which converts formyl-tetrahydrofolate to tetrahydrofolate, they may have different physiological effects in different types of cancer. Moreover, in different cancer types, the functions of metabolic enzymes may be diverse. Studying the mechanism of metabolic enzymes in different cancer types will promote the development of specific cancer therapies.

\section{Acknowledgements}

This study was supported in part by a Grant-in-Aid for Scientific Research from the Ministry of Education, Culture, Sports, Science and Technology; a Grant-in-Aid from the Third Comprehensive 10-year Strategy for Cancer Control, Ministry of Health, Labor and Welfare; a grant from the Kobayashi Cancer Research Foundation; a grant from the Princess Takamatsu Cancer Research Fund, Japan; a grant from the
National Institute of Biomedical Innovation; and a grant from the Osaka University Drug Discovery Funds. A.H. is a research fellow of the Japan Society for the Promotion of Science. Partial support was received from Taiho Pharmaceutical Co., Ltd. (H.I., J.K. and M.M.), Chugai Co., Ltd., Yakult Honsha Co., Ltd., Merck Co., Ltd., Takeda Science Foundation and Takeda Medical Research Foundation (M.K., M.M., N.N. and H.I.) through institutional endowments.

\section{References}

1. Brown JM and Giaccia AJ: The unique physiology of solid tumors: Opportunities (and problems) for cancer therapy. Cancer Res 58: 1408-1416, 1998.

2. Daşu A, Toma-Daşu I and Karlsson M: Theoretical simulation of tumour oxygenation and results from acute and chronic hypoxia. Phys Med Biol 48: 2829-2842, 2003.

3. Warburg O: On the origin of cancer cells. Science 123: 309-314, 1956.

4. Choi SW and Mason JB: Folate status: Effects on pathways of colorectal carcinogenesis. J Nutr 132 (Suppl): 2413S-2418S, 2002.

5. Duthie SJ and Hawdon A: DNA instability (strand breakage, uracil misincorporation, and defective repair) is increased by folic acid depletion in human lymphocytes in vitro. FASEB J 12: 1491-1497, 1998.

6. Widschwendter $M$ and Jones PA: DNA methylation and breast carcinogenesis. Oncogene 21: 5462-5482, 2002.

7. Pike ST, Rajendra R, Artzt K and Appling DR: Mitochondrial C1-tetrahydrofolate synthase (MTHFD1L) supports the flow of mitochondrial one-carbon units into the methyl cycle in embryos. J Biol Chem 285: 4612-4620, 2010.

8. Ventrella-Lucente LF, Unnikrishnan A, Pilling AB, Patel HV, Kushwaha D, Dombkowski AA, Schmelz EM, Cabelof DC and Heydari AR: Folate deficiency provides protection against colon carcinogenesis in DNA polymerase beta haploinsufficient mice. J Biol Chem 285: 19246-19258, 2010.

9. Jain M, Nilsson R, Sharma S, Madhusudhan N, Kitami T, Souza AL, Kafri R, Kirschner MW, Clish CB and Mootha VK: Metabolite profiling identifies a key role for glycine in rapid cancer cell proliferation. Science 336: 1040-1044, 2012.

10. Vazquez A, Tedeschi PM and Bertino JR: Overexpression of the mitochondrial folate and glycine-serine pathway: A new determinant of methotrexate selectivity in tumors. Cancer Res 73: 478-482, 2013.

11. Liu F, Liu Y, He C, Tao L, He X, Song H and Zhang G: Increased MTHFD2 expression is associated with poor prognosis in breast cancer. Tumour Biol 35: 8685-8690, 2014.

12. Díez-Villanueva A, Mallona I and Peinado MA: Wanderer, an interactive viewer to explore DNA methylation and gene expression data in human cancer. Epigenetics Chromatin 8: 22, 2015.

13. Gao J, Aksoy BA, Dogrusoz U, Dresdner G, Gross B, Sumer SO, Sun Y, Jacobsen A, Sinha R, Larsson E, et al: Integrative analysis of complex cancer genomics and clinical profiles using the cBioPortal. Sci Signal 6: pl1, 2013.

14. Cerami E, Gao J, Dogrusoz U, Gross BE, Sumer SO, Aksoy BA, Jacobsen A, Byrne CJ, Heuer ML, Larsson E, et al: The cBio cancer genomics portal: An open platform for exploring multidimensional cancer genomics data. Cancer Discov 2: 401-404, 2012.

15. Koseki J, Colvin H, Fukusumi T, Nishida N, Konno M, Kawamoto K, Tsunekuni K, Matsui H, Doki Y, Mori M, et al: Mathematical analysis predicts imbalanced IDH1/2 expression associates with 2 -HG-inactivating $\beta$-oxygenation pathway in colorectal cancer. Int J Oncol 46: 1181-1191, 2015.

16. Edge S, Byrd DR, Compton CC, Fritz AG, Greene FL and Trotti A (eds): AJCC Cancer Staging Handbook: From the AJCC Cancer Staging Manual. Springer, New York, 2011.

17. Miyo M, Yamamoto H, Konno M, Colvin H, Nishida N, Koseki J, Kawamoto K, Ogawa H, Hamabe A, Uemura M, et al: Tumour-suppressive function of SIRT4 in human colorectal cancer. Br J Cancer 113: 492-499, 2015.

18. Nilsson R, Jain M, Madhusudhan N, Sheppard NG, Strittmatter L, Kampf C, Huang J, Asplund A and Mootha VK: Metabolic enzyme expression highlights a key role for MTHFD2 and the mitochondrial folate pathway in cancer. Nat Commun 5: 3128, 2014. 
19. Dang L, White DW, Gross S, Bennett BD, Bittinger MA, Driggers EM, Fantin VR, Jang HG, Jin S, Keenan MC, et al: Cancer-associated IDH1 mutations produce 2-hydroxyglutarate. Nature 462: 739-744, 2009.

20. Jin S, Chen J, Chen L, Histen G, Lin Z, Gross S, Hixon J, Chen Y, Kung C, Chen Y, et al: ALDH2(E487K) mutation increases protein turnover and promotes murine hepatocarcinogenesis. Proc Natl Acad Sci USA 112: 9088-9093, 2015.

21. Lehtinen L, Ketola K, Mäkelä R, Mpindi JP, Viitala M, Kallioniemi $\mathrm{O}$ and Iljin K: High-throughput RNAi screening for novel modulators of vimentin expression identifies MTHFD2 as a regulator of breast cancer cell migration and invasion. Oncotarget 4: 48-63, 2013.
22. Kim D,Fiske BP,Birsoy K, Freinkman E, Kami K, Possemato RL, Chudnovsky Y, Pacold ME, Chen WW, Cantor JR, et al: SHMT2 drives glioma cell survival in ischaemia but imposes a dependence on glycine clearance. Nature 520: 363-367, 2015.

23. Ben-Sahra I, Hoxhaj G, Ricoult SJ, Asara JM and Manning BD: mTORC1 induces purine synthesis through control of the mitochondrial tetrahydrofolate cycle. Science 351: 728-733, 2016.

24. Csibi A, Fendt SM, Li C, Poulogiannis G, Choo AY, Chapski DJ, Jeong SM, Dempsey JM, Parkhitko A, Morrison T, et al: The mTORC1 pathway stimulates glutamine metabolism and cell proliferation by repressing SIRT4. Cell 153: 840-854, 2013. 